\title{
Platelet-rich plasma vs platelet-rich plasma plus hyaluronic acid for haemophilic knee arthropathy treatment
}

\author{
María Eulalia Landro, Carla Daffunchio, Guillermo Cambiaggi, Gustavo Galatro, Horacio Caviglia
}

From the Juan A. Fernández General Hospital. Orthopedic and Traumatology Department

Repeated joint bleeding leads to chronic synovitis, cartilage damage and bone alterations which result in haemophilic arthropathy and are associated with pain, functional impairment and poor quality of life. There are evidence that Hyaluronic Acid (HA) and Platelet-rich Plasma (PRP) have different mechanisms of action in the treatment of arthropathy for this reason we decided to use both components. The aim of this study is to compare, the efficacy, safety and duration of a single intra-articular injection of PRP against PRP+HA for pain, bleeding episodes and joint health, in the same patient with bilateral hemophilic knee arthropathy. Twenty-one men patients ( 42 knee joints) were treated with intraarticular injections of PRP or PRP+HA. All of them were haemophilia type $A$ severe. The mean age was 36.6 years (21-72). All patients were evaluated for: Haemophilia Joint Health Score (HJHS), pain (VAS), the number of bleeding episodes (BE) in the last 30 days, before treatment, at three and six months after treatment. Statistically significant improvement were shown for both knee joints at three and six months after treatment for VAS and $B E(P<0.00001)$. The HJHS score did not significantly improve for either knee in the 6-month period after injection. A single PRP or PRP+HA injection is safe and effective in treating haemophilic arthropathy of the knee for up to 6 months follow-up, reducing pain, bleeding episodes and delaying total knee arthroplasty.

Keywords: Knee arthropathy; haemophilia; platelet-rich plasma; hyaluronic acid.

No benefits or funds were received in support of this study. None of the authors have a conflict of interest.

\section{INTRODUCTION}

Repeated joint bleeding leads to chronic synovitis, cartilage damage and bone alterations which result in haemophilic arthropathy and are associated with limited range of motion (ROM), pain, muscle atrophy, functional impairment, and poor quality of life $(1,2)$.

Two therapies have been evaluated in this work for hemophilic arthropathy treatment: Hyaluronic Acid (HA) and platelet-rich plasma (PRP). In recent years, intra-articular injections with platelet-rich plasma and hyaluronic acid have shown long-term beneficial effects (3).

Hyaluronic Acid is a linear polysaccharide formed by repeating disaccharide units D-glucuronic acid and $\mathrm{N}$-acetylglucosamine (4), is the major part of the extracellular matrix of human tissues including the articular cartilage where HA is essential for

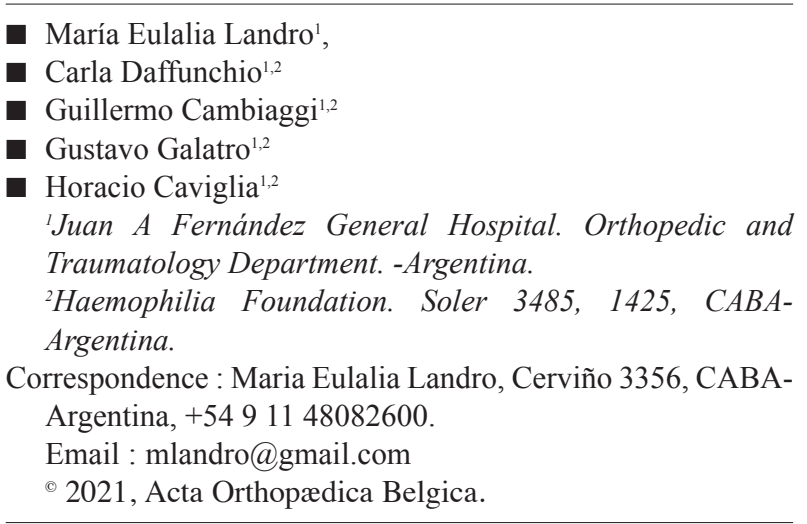


the visco-elastic and mechanical properties of the synovial fluid $(5,6)$.

The HA in synovial joints produce shock absorption on the joint surfaces and reduce pain as well also anti-inflammatory and chondroprotective effects (7-9).

In 2018, the Federal Register of United States published the intent to consider the appropriate classification of HA intra-articular products for pain treatment in osteoarthritis of the knee based on scientific evidence $(10,11)$.

Intra-articular injections of HA, restores synovial fluid viscoelasticity and mechanical properties by stimulating endogenous HA production from chondrocytes and sinoviocytes (5).

Palazzi et al. in 2002 first used intra-articular HA injections for haemophilic arthropathy and reported beneficial effects (12).

Intra-articular administration of PRP is a simple and accessible regenerative protocol based on the action of platelet-derived growth factors, and it is used for several pathologies. Intra-articular PRP injections are safe and minimally invasive procedure, effective in reducing pain and increasing joint function in patients diagnosed with knee osteoarthritis $(13,14)$.

In 2014, Teyssler et al. published the first experience using PRP for chronic synovitis treatment in hemophilia (15).

A combination of PRP and HA could prove to be more effective in treating knee arthropathy than either of the products alone.

The aim of this study is to compare, the efficacy, safety and duration of a single intra-articular injection of PRP against PRP + HA for pain, bleeding episodes and the Hemophilia Joint Health Score, in the same patient with bilateral hemophilic knee arthropathy.

\section{PATIENTS AND METHODS}

This study was approved by the institutional ethics committee protocol number 201823 and follows the declaration of Helsinki. Patients signed their informed consent to be part of the study.

Inclusion criteria: patients between 13 and 80 years-old with haemophilia A or B with bilateral

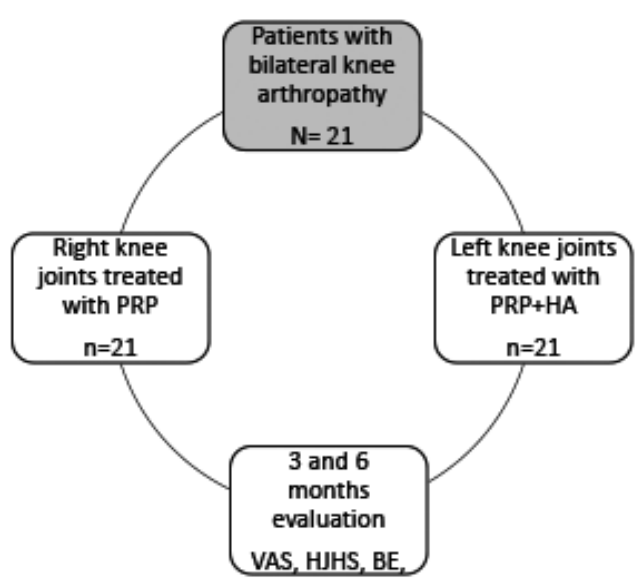

Fig. 1. - Study flow scheme.

knee arthropathy (I to IV Arnold-Hilgartner score) diagnosed by X-ray and clinical evaluation, who have not responded to six weeks of NSAID treatment and physical therapy. The study included both patients with and without synovitis.

Exclusion criteria: haemofílic arthropathy grade V Arnold-Hilgartner score, diagnosed by X-ray and clinical evaluation. No joint infection a year before treatment. Patients under corticosteroid treatment and who had previously received PRP or hyaluronic acid injections in knee joints.

Twenty-one men haemophilia type A severe patients with bilateral knee arthropathy (42 knee joints) were treated. Knee joints were intraarticularly injected. PRP was injected in right knee (RK) joints and PRP+HA was injected in left knee (LK) joints (Figure 1).

None of the patients had inhibitors. The mean age of the study population was 36.6 years (21-72). Left knee joints were grade III (11) and grade IV (10). Right knee joints were grade II (2), grade III (12), and grade IV (7).

All patients were evaluated for clinical outcome limiting the Hemophilia Joint Health Score (HJHS) for each knee joint, and for pain using the Vas scale (Visual Analogue Score). In addition, the number of bleeding episodes (BE) in the last 30 days were measured. All of these were evaluated before treatment, at three and six months after treatment.

PRP was obtained by extracting $10 \mathrm{ml}$ autologous peripheral venous blood, using the BD vacutainer ${ }^{\circledR}$ extraction kit and centrifuged at $200 \mathrm{G}$ for 8 minutes 
(Presvac ${ }^{\circledR}$ centrifuge, Bs. As.-Argentina). Five ml of the PRP was separated in a security cabinet and placed in a five $\mathrm{ml}$ syringe.

PRP+HA was prepared with a Cellular Matrix kit (Cellular Matrix A-CP HA Kit, Regen Lab® SA, Le Mont-sur-Lausanne, Switzerland) according to the operating instructions supplied with the kit. The device contains $2 \mathrm{ml}$ of natural, non-crosslinked, fermented HA $(1.550 \mathrm{kDa})$ at a concentration of $15 \mathrm{mg} / \mathrm{ml}$ that allows automated blood collection and blood component separation in a closed circuit. After centrifuging for five minutes in a Regen $\operatorname{Lab}{ }^{\circledR}$ centrifuge, $5 \mathrm{ml}$ of PRP+HA was obtained and placed in a five $\mathrm{ml}$ syringe.

After blood collection, coagulation factors were infused, according to the indications of the haematologist, before the injection to keep the factor level at $50 \%$ and infused again to maintain levels $=0>30 \%$ over the next three days.

After preparation, in the operating room, five $\mathrm{ml}$ of PRP or PRP $+\mathrm{HA}$ as appropriate, were injected into the corresponding affected knee joint. Joints were cleaned with $2 \%$ chlorhexidine and covered with a sterile drape. The PRP and the PRP+HA were injected with a 50/8 needle, assisted by ultrasound imaging.

After treatment, patients continued their previous factor therapy according to their hematologist's prescription.

The use of nonsteroidal anti-inflammatory medication was prohibited fifteen days before and after treatment.

Pain, bleeding episodes and HJHS for each knee were monitored at three and six- months after treatment by a blind evaluating physiotherapist, who does not know what treatment each knee received.

\section{Statistical Analysis}

The equality of variances was evaluated in both groups, accepting the null hypothesis at $95 \%$ reliability in all cases, and comparing the results before treatment and after treatment (at three and six months) in terms of its effect on VAS, BE and HJHS.

The difference in the mean result of the treatment applied was evaluated independently for each joint
Table I. - Visual Analogue Scale (VAS) and Bleeding Episode (BE) mean values, before treatment and three and six-months after treatment

\begin{tabular}{|l|c|c|c|c|}
\hline \multirow{2}{*}{ Data/Joint } & \multicolumn{2}{|c|}{$\begin{array}{c}\text { Right Knee } \\
\text { RK }\end{array}$} & \multicolumn{2}{c|}{$\begin{array}{c}\text { Left Knee } \\
\text { LK }\end{array}$} \\
\hline Variables & VAS & BE & VAS & BE \\
\hline Previous & 5.7 & 1.6 & 6.2 & 2.3 \\
\hline 3 months & 2.7 & 0.5 & 3.2 & 0.7 \\
\hline 6 months & 2.1 & 0.6 & 2.6 & 0.7 \\
\hline
\end{tabular}

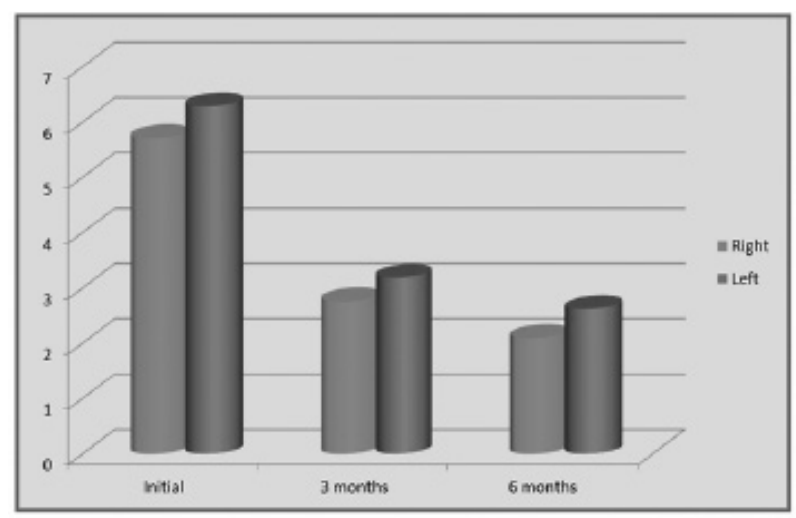

Fig. 2. - Visual Analogue Scale (VAS) mean values comparing both knee joints before treatment and after 3- and 6-month follow-up.

in terms of the three aspects on which the analysis was focused: VAS, BE and HJHS.

A p less than 0.05 is considered statistically significant.

\section{RESULTS}

Results show statistically significant improvement in the average scores, for both LK and RK joints at three and six months after treatment for VAS and $\mathrm{BE}(\mathrm{P}<0.00001)$ (Table I).

There were no statistical differences for VAS and $\mathrm{BE}$ at three- vs six-month follow-up.

There were no significant difference (95\% reliability) in the average results for both knee joints, which received different treatment in any of the parameters evaluated:

Visual analogue scale, RK vs LF, p value after three months treatment was 0.272 , and after six months treatment was 0.217 (Fig. 2). 


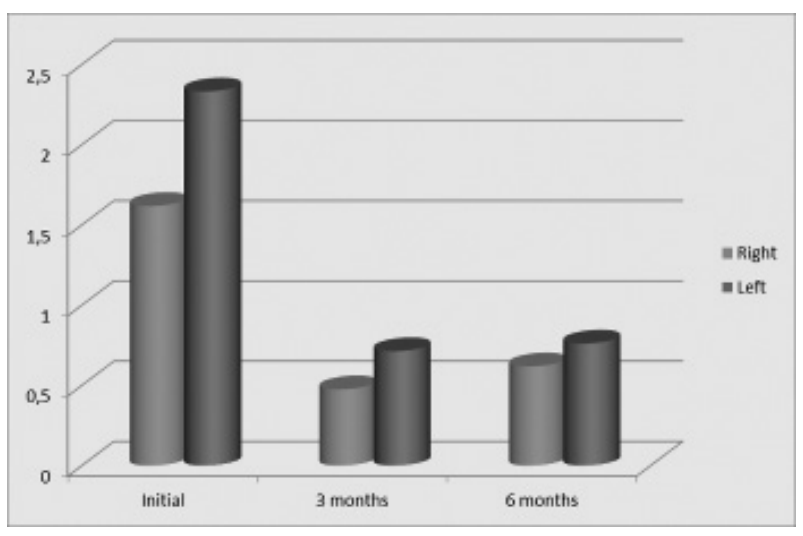

Fig. 3. - Bleeding Episodes (BE) mean values comparing both knees before treatment and after 3- and 6-months follow-up.

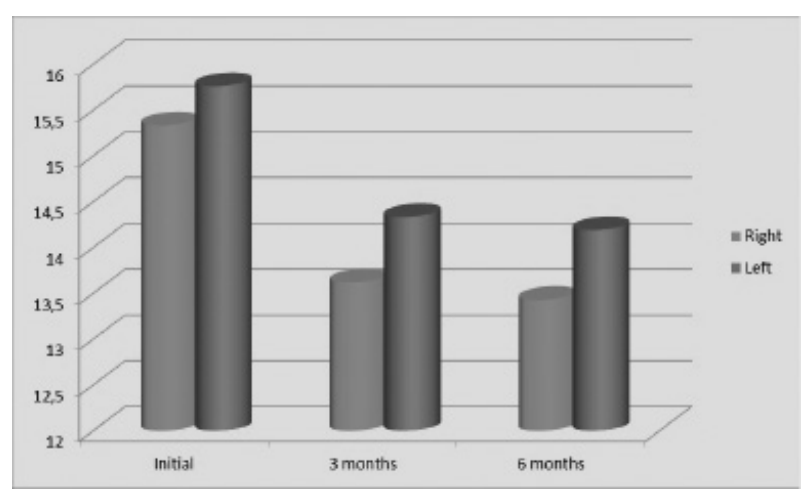

Fig. 4. - Heamophilia joint Health Score (HJHS) mean values comparing both knees before treatment and after 3- and 6-months follow-up.

Bleeding episodes, RK vs LK, $\mathrm{p}$ values after three months treatment was 0.417 , and for six months treatment was 0.282 (Fig. 3).

The HJHS score did not significantly improve for either knee in the 6-month period after injection (Table II).

The HJHS was not statistically significant for RK vs LK at 3 months after treatment p: 0.256 and after 6 months treatment p: 0.247 (Fig. 4).

Improvement in both stiffness and joint crepitant was greater in left knee joints treated with PRP+HA.

\section{DISCUSSION}

Hemophilic arthropathy produces cartilage damage and subchondral bone due to the deleterious effect of the blood, leading to apoptosis of chondrocytes, that is an irreversible process (16).
Table II. - Heamophilia Joint Health score (HJHS) mean values before treatment, three and six-months after treatment

\begin{tabular}{|l|c|c|}
\hline HJHS & $\begin{array}{c}\text { Right Knee } \\
\text { RK }\end{array}$ & $\begin{array}{c}\text { Left knee } \\
\text { LK }\end{array}$ \\
\hline Previous & 15.3 & 15.7 \\
\hline 3 months & 13.6 & 14.3 \\
\hline 6 months & 13.4 & 14.2 \\
\hline
\end{tabular}

Non-invasive treatments that were carried out in the damaged joint of the PWH, only seek to avoid further deterioration of the compromised joint.

The effectiveness of intra-articular HA injections in hemophilic arthropathy has been demonstrated in several publications.

The first experience carried out with HA in patients with hemophilia was published by Fernandez Pallazzi et al. in 2002 (12). They report pre-liminary results using hyaluronic acid $\left(\right.$ Synvisc $\left.^{\circledR}\right)$ in 29 grade III joints (chronic arthropathy, axial deformities and muscle atrophy) in 25 patients with haemophilia (23 type A and 2 type B) including: 17 knees, six shoulders, four ankles, one elbow and one hip. Patients were injected once a week for three weeks, with a mean follow-up of 10.5 months. Patients reported complete pain relief in $13.7 \%$ of the injected joints and partial relief in $62 \%$, resulting in a total of excellent and good results in $75 \%$. Only $10.3 \%$ reported poor results (no improvement at all and needing another procedure). Fernandez Palazzi reported satisfactory results and recommends HA for hemophilic arthritis, due to physiological properties, against the use of articular long-standing corticosteroids that could produce cartilage damage (12).

Wallny et al. published their experience using HA in patients with hemophilic arthropathy and proved the effectiveness of intra-articular polysaccharide in the medium term, since their follow-up was 26 months. In this study, 20 patients with 21 affected joints were evaluated and treated, with five weeks intra-articular injection with HA. No differences were detected in MRIs. After three months, 14 of 20 patients showed improved walking distance, stair-climbing and pain relief. After 26 months ten patients are still showing improvement for up 
to 31 months follow-up. They recommend HA for haemophilic arthropathy of the knee when regular conservative therapy has failed (17).

Carulli et al. published their long-term experience, using intra-articular HA injections in patients with hemophilic arthropathy of the knee. The study included 27 patients treated with at least two cycles of injections of hyaluronan and evaluated with VAS, Short Form-36 (SF-36), World Federation of Haemophilia score (WFH), Pettersson score, and WOMAC, with a seven-year follow-up. All patients showed improvement in pain relief and functional recovery without any complications. Only five patients required total knee arthroplasty due to persistent pain or functional limitation (18).

PRP has proven to be effective in the treatment of synovitis in PWH, after one-year follow-up, reducing the number of bleeding episodes, pain and clinically improving HJHS (19).

$\mathrm{Li}$ et al. have demonstrated the effectiveness of PRP in hemophilic arthropathy, in the short term. This study compared the efficacy of intra-articular PRP vs HA injections for knee joints. They treated 22 knees in an open-label and observer-blind study. Eleven patients were treated with a single intraarticular injection of PRP, and the other eleven received five consecutive weekly intra-articular injections of HA. Evaluations were performed for: VAS, WOMAC and synovial change observed by ultrasonography. Both treatments were found to be effective in reducing pain and improving functional status of the knee. There were no significant differences between PRP and HA groups in VAS, SF-36 score, WOMAC score, ROM, number of hemarthrosis, hyperemia and thickness of synovium. The study showed that a single intra-articular PRP injection was effective in treating haemophilic arthropathy of the knee joint for a period of at least six months (6).

In our study we compared the effectiveness of PRP or PRP+HA, in both knees of the same patient, who suffered bilaterally hemophilic arthropathy. This clinical model avoids the variability that exists between patients.

Most of the published clinical studies, using intraarticular HA to treat knee arthropathy, use three to five injections of the polysaccharide $(17,18)$.
In this clinical research study, an intra-articular PRP injection was compared against one injection of PRP+HA, in both knees of the same patient, in order to observe clinical result obtained by the addition of hyaluronic acid to PRP. We decided to use a commercial kit (Cellular Matrix ${ }^{\circledR}$ ), since it allows both products to be applied together as an emulsion, since both products have different viscosity. This commercial kit suggests a series of three session of injections with one-month intervals. In this study we compare a single injection to limit the variables of the study.

The fact that PRP can be prepared in the laboratory without a commercial kit which makes it more accessible, from an economic point of view, than $\mathrm{PRP}+\mathrm{HA}$ is important. However, as it is an open procedure, it requires operator trained manipulation, and laboratory equipment. Contamination risks due to sample manipulation must be taken into account.

Closed commercial kits like the one for PRP $+\mathrm{HA}$, despite its cost, allow us to obtain a quality, safe and standardized product that does not depend on sample manipulation.

This combination of $\mathrm{PRP}+\mathrm{HA}$, may provide pain relief and functional improvement in patients with advanced knee arthropathy, and delay joint deterioration (20).

The parameters evaluated in this study were higher in LK joints than those of RK joints. This is due to the fact that left knees presented higher degrees of arthropathy (III and IV) and synovitis than right knees (arthropathy degree II, III and IV).

This study shows statistically significant improvement in the average scores, for both LK and RK joints at three and six months after treatment for VAS and BE $(\mathrm{P}<0.00001)$. These results show early improvements and these benefits did not change after three months treatment. Pain improvement was observed in both groups, and was sustained up to six months.

In addition to joint lubrication there are evidence showing that HA and PRP have different and/ or complementary mechanisms of action in the treatment of haemophilic arthropathy, intraarticular injection of PRP $+\mathrm{HA}$ potentially reduces chondrocyte apoptosis, increases chondrocyte proliferation, up regulates proteoglycan and glyco- 
saminoglycan synthesis, down regulates degradative enzyme, inhibits catabolic cytokine suppression, and relieves pain (21).

There were no statistical differences between treatments, both are effective for hemophilic knee arthropathy. HJHS scores also improved, but it has not been statistically significant. This is probably due to that the HJHS evaluates multiples variables to obtain the status of joint health, including: swelling, duration of swelling, muscle atrophy, crepitus of motion, range of motion, pain, strength, and gait. It is an internationally validated physical examination assessment tool (22). Patients included in this study reported significant less joint stiffness and the absence of joint crepitant after PRP $+\mathrm{HA}$ treatment in left knee joints.

However, patients reported, at three- and sixmonths controls, the improvement of these two symptoms that had a great impact in their daily lives.

No side effects were observed in this study associated with intra-articular injections due to the autologous nature and composition of PRP and $\mathrm{PRP}+\mathrm{HA}$,

Several studies have demonstrated the presence of reactive oxygen species (ROS) in the joints of patients with osteoarthritis $(23,24)$. There is evidence for an age-related imbalance in ROS production relative to the antioxidant capacity of chondrocytes that plays a role in cartilage degradation as well as chondrocyte apoptosis $(25,26)$. High levels of ROS cause oxidative-damage, and ROS acts as a second messenger, mediating intracellular signaling events that down regulate gene expression such as the expression of matrix degrading enzymes (27).

Based on these findings, preliminary in vitro and animal-based studies have postulated that intraarticular injection of iron-chelating drugs and ROS scavengers could be used to treat haemophilic joint damage (28).

In 1973, McCord et al. showed, in vitro, that hyaluronic acid was susceptible to degradation by ROS (29). Eliminators of reactive oxygen species have also been reported to inhibit hyaluronic acid degradation by ROS (30).

Dahl et al. recently reported that patients with rheumatoid arthritis had lower HA concentrations in synovial fluids. These findings support the hypothesis that ROS are responsible for the accelerated degradation of HA in rheumatoid joints $(29,31)$.

All these results suggest that PRP and HA could have complementary beneficial anti-inflammatory and anabolic effects on joints. A combination of the two might produce better results than either PRP or HA alone for the treatment of haemophilic arthropathy (21).

The combination of HA and plasma-rich in growth factors could arrest the degeneration process. This positive biological interaction could improve the efficacy of treatment due to stimulation and inflammatory response (32).

Using PRP + HA together can inhibit inflammation and improve chondrogenesis more efficiently than separately. Our previous, in vitro, experimental study demonstrated that oxidation of $\mathrm{Hb}$ (hemoglobin) to $\mathrm{MeHb}$ was completely inhibited either by PRP, PPP or albumin as a physiologic iron chelator and ROS scavenger, it is possible that plasma albumin contributes to the anti-oxidative role of PRP. This could explain the therapeutic effect of intra-articular joint injection of PRP in patients with haemophilia (31). It is also feasible that the albumin applied with the PRP, reduces the amount of intra-articular ROS and prevents the degradation of hyaluronic acid. PRP associated with HA would enhance the action of the polysaccharide injected intra-articularly. Significant less joint stiffness and the absence of joint crepitant after PRP + HA treatment in left knee joints were shown.

The limitations of this study include small samples due to the difficulty of recruiting patients with bilateral knee arthropathy with similar degrees of joint damage. The follow-up period of 6 months is another limitation of the present study.

The strength of this study is that it compares two treatments for hemophilic arthropathy of the knee in a same patient, removing variability between patients.

We suggest that a single PRP or PRP+HA injection is safe and effective in treating haemophilic arthropathy of the knee for up to 6 months follow-up. While no difference between PRP and PRP+HA knee joints were found, both groups demonstrated decreases in pain and bleeding 
episodes. Both ttreatments not only reduced pain, bleeding episodes and stiffness, it also improved knee function in patients with grade II, III and IV arthropathy, providing a good option for temporary management and delaying total knee arthroplasty. Based on scientific evidence, PRP and HA seem to share some therapeutic effects and could have complementary beneficial anti-inflammatory and anabolic effects on joints with haemophilic arthropathy. Further investigation is needed to standardize its application.

\section{REFERENCES}

1. Srivastava A, Brewer AK, Mauser-Bunschoten EP, Key NS, Kitchen S, Llinas A, et al. Guidelines for the management of hemophilia. Haemophilia 2013; 19(1): $1-47$.

2. Kurien T, Arendt-Nielsen L, Petersen KK, GravenNielsen T, Scammell BE. Preoperative neuropathic pain-like symptoms and central pain mechanisms in knee osteoarthritis predicts poor outcome 6 months after total knee replacement surgery. J Pain 2018; 19(11): 1329-1341.

3. Huebner K, Frank RM, Getgood A. Ortho-biologics for osteoarthritis. Clin Sports Med 2019; 38(1): 123-141.

4. Vasi AM, Popa M, Butnaru M, Dodi G, Verestiuc L. Chemical Functionalization of Hyaluronic Acid for Drug Delivery Applications. Materials Science and Engineering 2014; 38: 177-185.

5. Qipeng Wu, Xuefen Luo, Yuan Xiong, Guohui Liu, Junwen Wang, Xi Chen, et al. Platelet-rich plasma versus hyaluronic acid in knee osteoarthritis: A meta-analysis with the consistent ratio of injection. J Orthop Surg 2020; 28(1) $1-9$.

6. Tsung-Ying Li, Yung-Tsan Wu, Liang-Cheng Chen, Shin-Nan Cheng, Ru-Yu Pan. An exploratory comparison of single intra-articular injection of platelet-rich plasma vs hyaluronic acid in treatment of haemophilic arthropathy of the knee. Haemophilia 2019; 25(3): 484-492.

7. Strauss E, Hart J, Miller M, Altman R, Rosen J. Hyaluronic Acid Viscosupplementation and Osteoarthritis: Current Uses and Future Directions. Am J Sports Med 2009; 37: 1636-1644.

8. Altman RD, Manjoo A, Fierlinger A, Niazi F, Nicholls M. The Mechanism of Action for Hyaluronic Acid Treatment in the Osteoarthritic Knee: A Systematic Review. BMC Musculoskeletal Disorders 2015; 16: 321.

9. Moreland LW. Intra-Articular Hyaluronan (hyaluronic acid) and Hylans for the Treatment of Osteoarthritis: Mechanisms of Action. Arthritis Res Therapy 2003; 5(2): 54-67.

10. Helfet A. The Physical Properties of Synovial Fluid and the Special Role of Hyaluronic Acid. In Disorders of the
Knee, Philadelphia: Lippincott Company.1974; 63-75.

11. Jahn S, Seror J, Klein J. Lubrication of Articular Cartilage. Annual Review of Biomedical Engineering 2016; 18: 235258.

12. Fernández-Palazzi F, Viso R, Boadas A, Ruiz-Sáez A, Caviglia H, De Bosch NB. Intra-articular hyaluronic acid in the treatment of haemophilic chronic arthropathy. Haemophilia 2002; 8(3): 375-381.

13. Vaquerizo V, Plasencia MÁ, Arribas I, Seijas R, Padilla S, Orive G. et al. Comparison of intra-articular injections of plasma rich in growth factors (PRGF-Endoret) versus durolane hyaluronic acid in the treatment of patients with symptomatic osteoarthritis: a randomized controlled trial. Arthroscopy 2013; 29(10):1635-1643.

14. Shen L, Yuan T, Chen S, Xie X, Zhang C. The temporal effect of platelet-rich plasma on pain and physical function in the treatment of knee osteoarthritis: systematic review and meta-analysis of randomized controlled trials. J Orthop Surg 2017; 12(1): 16-27.

15. Teyssler K, Kolostova K, Bobek V. The impact of plateletrich plasma on chronic synovitis in hemophilia. Acta Orthop Belg. 2014; 80(1): 11-7.

16. Knobe K, Berntorp E. Haemophilia and Joint Disease: Pathophysiology, Evaluation, and Management. J Comorb 2011; 1: 51-59.

17. Wallny T, Brackmann H, Semper H, Schumpe G, Effenberger W, Heû L. et al. Intra-articular hyaluronic acid in the treatment of haemophilic arthropathy of the knee. Clinical, radiological and sonographical assessment Haemophilia 2000; 6(5): 566-70.

18. Carulli C, Matassi F, Civinini R, Morfini M, Tani M, Innocenti M. Intra-articular injections of hyaluronic acid induce positive clinical effects in knees of patients affected by haemophilic arthropathy. Knee 2013; 20(1): 36-39.

19. Landro ME, Daffunchio C, Cambiaggi G, Galatro G, Salgado P, Honnorat E. et al. Platelet Rich Plasma Intra Articular Injection for Chronic Synovitis Treatment in Patients with Haemophilia One Year Follow Up. Biomed J Sci \& Tech Res 2019; 2(5): 9572-9575.

20. Chen SH, Kuan TS, Kao MJ, Wu WT, Chou LW. Clinical effectiveness in severe knee osteoarthritis after intraarticular platelet-rich plasma therapy in association with hyaluronic acid injection: three case reports. Clin Interv Aging 2016 Selt 8; 11, 1213-1219.

21. Chen WH, Lo WC, Hsu WC, Wei HJ, Liu HY, Lee CH. et al. Synergistic anabolic actions of hyaluronic acid and platelet-rich plasma on cartilage regeneration in osteoarthritis therapy. Biomaterials 2014; 35(36): 95999607.

22. Hilliard P, Funk S, Zourikian N, Bergstrom BM, Bradley CS, Mc Limont M, et al. Hemophilia joint health score reliability study. Haemophilia 2006; 12: 518-25.

23. Karan A, Karan MA, Vural P, Erten N, Tascioglu C, Aksoy C, et al. Synovial fluid nitric oxide levels in patients with knee osteoarthritis. Clin. Rheumatol 2003, 22(6): 397399. 
24. Lepetsos P, Papavassiliou A. ROS/oxidative stress signaling in osteoarthritis. Biochimica et Biophysica Acta (BBA) - Molecular Basis of Disease 2016; 1862(4): 576591.

25. Altay MA, Erturk C, Bilge A, Yapti M, Levent A, Aksoy N. Evaluation of prolidase activity and oxidative status in patients with knee osteoarthritis: relationships with radiographic severity and clinical parameters. Rheumatol Int 2015 Oct; 35(10): 1725-31.

26. Davies CM, Guilak F, Weinberg JB, Fermor B. Reactive nitrogen and oxygen species in interleukin-1-mediated DNA damage associated with osteoarthritis Osteoarthr Cartil 2008; 16(5): 624-630.

27. Bolduc J, Collins $\mathbf{J}$ and Loeser RF. Reactive Oxygen Species, Aging and Articular Cartilage Homeostasis. Free Radic Biol Med 2019; 132: 73-82.

28. Nieuwenhuizen L, Roosendaal G, Mastbergen SC, Coeleveld K, Biesma DH, Lafeberet F. et al. Deferasirox limits cartilage damage following haemarthrosis in haemophilic mice. Thromb Haemost 2014; 112(5): 1044-1050.

29. McCord JM. Free radicals and inflammation: protection of synovial fluid by superoxide dismutase. Sciences 1974; 4150(185): 529-531.

30. Blake DR, Hall ND, Treby DA. Protection against superoxide and hydrogen peroxide in the synovial fluid of rheumatoid patients. Clin Sci 1981; 61(4): 483-486.

31. Caviglia H, Daffunchio C, Galatro G, Cambiaggi G, Oneto P, Douglas Price Al, et al. Inhibition of Fenton reaction is a novel mechanism to explain the therapeutic effect of intra-articular injection of PRP in patients with chronic haemophilic synovitis. Haemophilia 2020; 26(4): 187-193.

32. Andia I, Maffulli N. Platelet-rich plasma for managing pain and inflammation in osteoarthritis. Nat. Rev. Rheumatol 2013; 9(12): 721-730. 